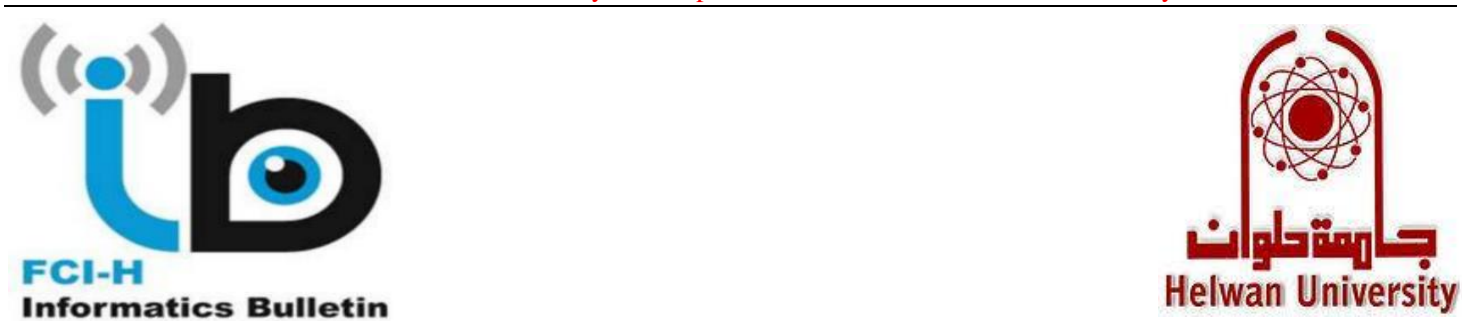

\title{
Adaptive Approach for Intelligent Web to Enhance Business Intelligence Applications
}

\author{
Yehia Helmy ${ }^{a}$, Ayman E. Khedr ${ }^{b}$, Shrief kolief ${ }^{a,}$ Eman Haggag ${ }^{a^{*}}$ \\ ${ }^{a}$ Faculty of Computers and Information, Helwan University, Egypt. \\ ${ }^{b}$ Faculty of Computers and Information Technology, Future University in Egypt, Egypt.
}

\section{KEYWORDS}

Web Intelligence (WI), Business Intelligence (BI), Recommender System (RS), Web Opinion Mining (WOM), Web Usage Mining (WUM).

\section{Introduction}

Business intelligence (BI) is a perfect way to transform data into interactive information that could lead to improve the processes of decision making at all levels of management (Popovič, et al, 2012). Companies use BI to detect significant events and identify business trends to adapt quickly to changes in their environment (Popovic et al., 2012).

\begin{abstract}
A B S T R A C T
The World Wide Web (WWW) has grown quickly in the past two decades from a small research community to the biggest and most popular infrastructure for communication, information dissemination, search, social interaction and commerce. The continuous growth in size and use of the WWW creates a need for methods to process these wicked volumes of data. Web Intelligence (WI), as a research direction, has a broad agenda to deal with the issues that arise around the WWW phenomenon. WI corresponds to research and development to explore the fundamental roles, artificial intelligence and advanced information technology on the web-empowered systems, services, and activities. In this context, WI is concerning of mining in web data and user behaviors. This creates a demand for using mining technologies to search large volume of data for gaining hidden knowledge. This hidden knowledge helps in gaining competitive advantages, better customers' relationships, and even fraud detection. Achieving the intelligence to the web enhances Business Intelligence (BI) for the enterprises. Web Usage Mining (WUM) and Web Opinion Mining (WOM) are considered a leading mining approaches in mining the user behavior and reviews. Most of previous studies depended in building intelligent web on mining and analyzing user's profiles or opinions separately. This becomes not fair enough and cause of limitations. This limitations could be narrowed if both of user preferences and opinions are considered in building recommendations. The paper proposes a framework for achieving the intelligence via using WUM and WOM. The proposed framework would contribute in solving the problem. The paper also surveys the background of using the intelligent web as an approach to enhance the Business Intelligence (BI) applications.
\end{abstract}

However, the implementation of business intelligence is not easy because of data complexity (Ranjan, 2009). Recently, WI is a central factor in BI success. As web is the main and the most important way to communicate between customer and the enterprise. WI helps enterprise to know exactly what customer need in what time. This knowledge is the base stone for the decisions that affect the performance of the overall business. Gaining accurate and precise knowledge is the corner stone of succeeding in achieving the BI.

*corresponding author. Tel.: 01005668123

E-mail address: emanhaggag141278sams@gmail.com 
The paper proposes a framework for achieving the WI via using WUM and WOM. The paper is organized as follow: background, literature survey and the proposed framework. The background part revises the WI and BI concepts and theories followed by a review for the opinion mining and Web Usage Mining (WUM)). A mention for Recommender System (RS) will also be given as it is one of the important topics in WI. The literature survey part explores the most recent researches in the fields of Web Intelligence (WI), Business Intelligence (BI), Recommender System (RS), opinion mining and Web Usage Mining (WUM). This would show the positive and negative aspects, as well as realizing the common work and issues. The aim of the literature survey part is to serve as a compass for researchers who are concerning of WI and BI field. Finally the proposed framework and conclusions.

\subsection{Web Intelligence (WI)}

Intelligence has been defined in many different ways including as one's capacity for logic, understanding, self-awareness, learning, emotional knowledge, planning, creativity and problem solving (Yao, et al, 2001)). It could be more described as the ability to perceive information and to retain it as knowledge to be applied towards adaptive behaviors within an environment or context (Camacho, 2013).

There are three components that need to be happened to harness community intelligence which are: Concede users to interact, learn about your users in aggregate and personalize content using user interaction data and aggregate data (Camacho, 2013).

\subsubsection{Web Intelligence Concept}

Web intelligence is about applying artificial intelligence and information technology techniques on the Web to create novel, adaptive and smarter web-based products, services and frameworks (Yao et al., 2001).

\subsubsection{Web Intelligence Levels}

The WI levels (Mithaiwala, \& Jariwala, 2016) could be thought as in fig 1.

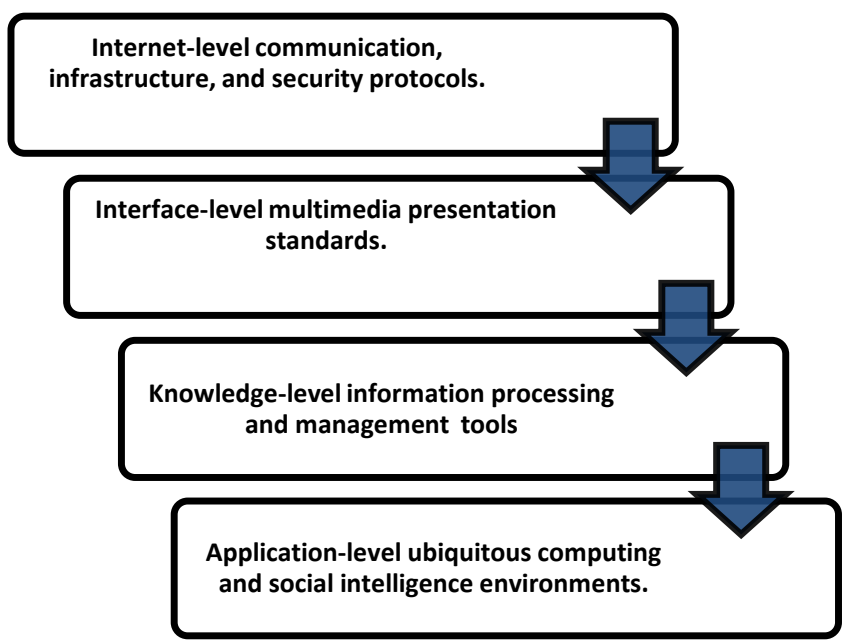

Fig1.WI Levels

\subsubsection{Elements Required to Build Web Intelligence Applications}

A according to the WI concepts and theories, three elements are determined and needed to build web intelligence applications which are (Ekstrand, et al, 2011; Marmanis \& Babenko, 2009)

- Aggregated Content: means a large amount of data pertinent to a specific application.

- $\quad$ Reference Structures: These structures provide one or more structural and semantic interpretations of the content.

- Algorithms: This refers to a layer of modules that allows the application to harness the information, which is hidden in the data, and use it for the purpose of abstraction (generalization), prediction, and (eventually) improved interaction with its users.

The previous elements are called the intelligence triangle and is illustrated as in figure 2

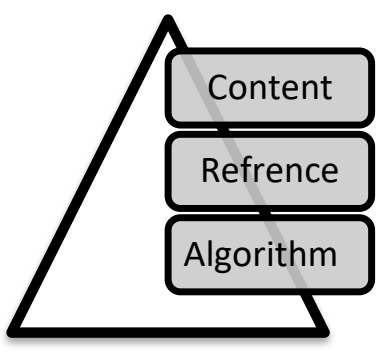

Fig2. Intelligence Triangle

\subsection{Business Intelligence (BI)}

Business intelligence is not business as usual. It's about making better decisions easier and making them more quickly (Ranjan, 2009). Business data is increasing exponentially. In the age of the information competition, every one need to be able to make better decisions faster. BI is getting the right data, discover its power, and share its value (Reinschmidt \& Francoise, 2000). Business Intelligence is the application gainning the right information and delivering it to the right user at the right time to enforce the decision-making process (Laursen \& Thorlund, 2016). BI could be thought as the processes, operations, techniques, technologies and tools needed to turn data into information and information into knowledge and knowledge into plans that drive profitable business action (Leat, 2007). BI umbrella includes data warehousing, business analytics and knowledge management (Leat, 2007).

\subsubsection{BI Data Sources}

BI sources could be (Ranjan, 2009):

- Relational databases.

- Operational databases.

- Historical data.

- External data.

- Information from the already existing data warehouse environment.

- Information from the already existing data warehouse environment.

- Unstructured information, such as plain text files or pictures. 


\subsubsection{The Development Process}

BI project has to deal with three tasks: Infrastructure, Data and Application (Reinschmidt \& Francoise, 2000):

- Infrastructure: preparing and managing the technical basis for the BI environment.

- Data: handling data requirements and business rules. Like data accessing, mapping, derivation, transformation.

- Application: collecting the business requirements, designing the model, and implement, visualize, and publicity the analysis results.

\subsection{Recommender System (RS)}

Recommender systems is concerning about finding the relevant items to customers to gain user satisfaction (Dong \& Smyth, 2016). a recommender system concerning about what the user want and what is the pros and cons of products or services (Dong \& Smyth, 2016). Recommender systems are information selector systems that manage the problem of information overload by concern and select the required information fragment out of large amount of automatic data generated with respect to user's interest (Sun, Kong, \& Ye, 2005).

\subsubsection{Recommender System Classification (RS)}

Categorizing RS differs according to the base of classification. It could be classified based on the information that RS uses (Rao, 2008). Fig 3 shows RS classification based on information.

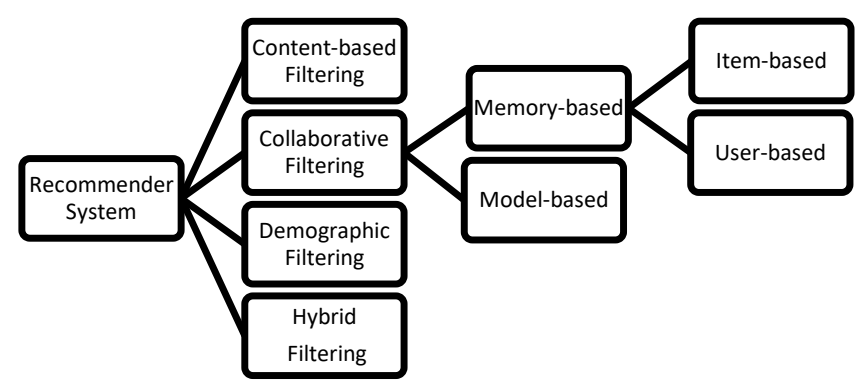

Fig 3. Recommender System Based on Information

Another classification is based on the filtering algorithm that the RS uses (Adomavicius \& Tuzhilin, 2005; Bobadilla, et al, 2013; Candillier, et al, 2007; Ekstrand, et al, 2011). Fig 4 shows the RS classification based on filtering algorithm.

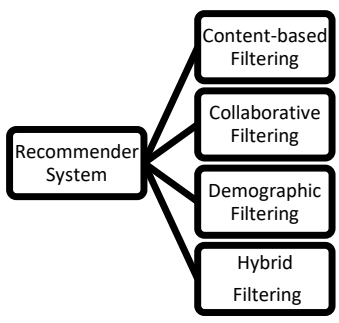

Fig 4. Recommender System Based on Filtering Algorithm
- Content-Based Filtering (CBF): tries to recommend items to the active user similar to those rated positively in the past. It is based on the concept that items with similar attributes will be rated similarly (Bobadilla et al., 2013; Lang, 1995; Paulson \& Tzanavari, 2003; Van Meteren \& Van Someren, 2000).

- Collaborative Filtering (CF): allows users to give ratings about a set of elements (e.g. videos, songs, films, etc. in a CF based website) in such a way that when enough information is stored on the system, recommendations to each user could be introduced based on information provided by those users which are considered to have the most in common with them (Adomavicius \& Tuzhilin, 2005; Bobadilla et al., 2013; Candillier et al., 2007).

- Demographic Filtering: is justified on the principle that individuals with certain common personal attributes (sex, age, country, etc.) will also have common interests (Bobadilla et al., 2013; Krulwich, 1997; Pazzani, 1999; Porcel, et al, 2012).

- Hybrid Filtering: Commonly uses a combination of CF with demographic filtering or $\mathrm{CF}$ with content-based filtering to exploit merits of each one of these techniques (Bobadilla et al., 2013; Burke, 2002; Porcel et al., 2012).

A widely accepted taxonomy divides recommendation methods into memory-based and model- based method (Bobadilla et al., 2013; Candillier et al., 2007; Sun, X., Kong, \& ye, 2005; Symeonidis, et al, 2009). Memory-based methods defined as methods that act only on the matrix of user ratings for items .while Model-based methods use RS information to create a model that generates the recommendations (Bobadilla et al., 2013; Candillier et al., 2007; Sun et al.,2005; Symeonidis et al,2009 ).

\subsection{Web Opinion Mining (WOM)}

WOM or Opinion mining is the use of natural language processing, text analysis and computational linguistics to identify and extract subjective information in source materials (Ahuja, 2017). Opinion Mining is concerning about analyzing people's opinion, sentiments, evaluations, attitudes and emotions from written text (Dhokrat, et al, 2015) .Opinion mining classifies the text to subjective or objective . Subjective means that the text contains/bears opinion content. Objective is a fact or general information rather than a view of some thing (Liu, 2012).

\subsubsection{Opinion Components}

Opinion could be categorized into three main components which are (Sharma, et al, 2014):

- Opinion Holder: is the holder for opinion; it may be a person or an organization that write the opinion.

- Opinion Object: is an object on which the opinion holder is expressing the opinion.

- Opinion Orientation: determines whether the opinion of an opinion holder about an object is positive, negative or neutral.

Fig 5 shows the opinion components. 


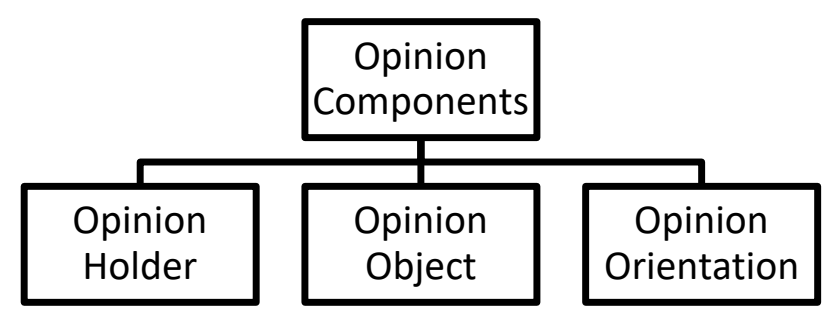

Fig 5. Opinion Mining Components

\subsubsection{Different Levels of Opinion Mining}

Opinion could be mined at three levels (Liu, 2012).

- Document level: shows the general attitude from the whole text.

- Sentence level: extracts the direction for the attitude (positive, negative or neutral) from each sentence.

- Entity and Aspect level: also called feature level, depends on extracting the features from opinion and generate a summarization.

\subsection{Web Usage Mining (WUM)}

WUM is getting knowledge from mining the Web data (Gonsalves, 2015). This knowledge could help in understanding and serving better the needs of the web based application (Neelima, \& Rodda, 2015).

\subsubsection{WUM Process}

WUM process contains four stages which are (Liu, et al, 2011):

- Web Usage Data Collection: Web usage data are gathered from various sources by using different techniques that allow to attain efficient collections of user data.

- Web Usage Data Preprocessing: The collected Web usage data are preprocessed to obtain data expressed in a form that is suitable to be analyzed in the next steps.

- Web Usage Pattern Discovery: The available usage data are analyzed to mine significant correlations between data and discover usage patterns, reflecting the behavior and the interests of users. Typically, in this stage learning methods, such as clustering, association rule discovery and sequential pattern discovery are applied to automate the process of pattern discovery.

- Application of Web Usage Patterns: The extracted knowledge expressed in terms of usage patterns is analyzed and evaluated to be exploited to implement the effective functionalities of the considered application such as recommendation engines, visualization tools, Web analytics and report generation tools.

\subsubsection{Association Rule Mining}

The most straightforward technique for pattern discovery in WUM is mining association rules. Association rule tries to find significant associations among groups of pages which frequently appear in user sessions (Agrawal, et al, 1993). Most famous algorithm in association is
Apriori (Agrawal, \& Srikant, 1994). Apriori is able to generate frequent item sets. starting from the frequent item sets, association rules which satisfy a minimum confidence threshold are generated (Ouertani, \& Alawadh, 2017. An association rule could be expressed in the following form: $X \Rightarrow(Y-X)$. Where $X$ and $Y$ are frequent item sets and $X \subset Y$. Item sets $X$ and $(Y-X)$ respectively represent the antecedent and the consequence of the rule. The generated association rules are valid only if the measure of confidence results will be equal or greater than an established minimal threshold (Agrawal et al., 1993).

\section{Literature Survey}

The tremendous popularity of web is attracting the attention of the industry to take profit from the massive availability of sentiment data, which has a high value for Business Intelligence (BI) (Mithaiwala \& Jariwala, 2016).

Web sites are seen as big data in the sense that they provide a massive amount of online reviews, user profiles and ratings. This data could be collected and analyzed to explore the hidden knowledge which help in improving the decision making process. The online behavior that exists today in the Web represents new sources of information. Recently, the automated discovery or mining of consumer opinions from these sources besides mining the user profiles have great importance for marketing intelligence and product benchmarking ( Marmanis, \& Babenko, 2009).

The following sections show some efforts and researches in using web intelligence via opinion mining and user profile in enhancing BI applications.

Funk et. al. (2017) employed linguistic information that is used by a supervised machine learning system to categorize text by rating (good or bad from 1 to 5). The approach developed a system to classify each text based on qualitative variable category from an ontology especially for BI. Funk et. al. (2017) indicated that it could be successfully classifying short texts. This could be done by using machine learning based on quick and simple analysis in rating with a standard NLP and without relying on predefined lists of opinion words. The approach provided an accurate picture of business entity.it does not use any predefined word lists or specialized lexical. The approach allow machine learning to infer the values of words implicitly from the training data.

Scolz et. al. (2017) proposed a system to help support consumers decision processes in e-commerce shopping tasks. The proposed system depended on a new method based on a product configuration process. The proposed method has been compared with two other methods which are Swing Ranking Based -Conjoint analysis and Trade Off. Results showed that the proposed method perform better and faster than other two compared methods.

Zhu et. al. (2017) investigated intellectual structure of the domain of opinion mining. The approach used topic search citation expansion and patent search. It applied a dual map overlay and a visual analytic system based on networks of co-cited documents to investigate epistemological characteristic of the domain structure of a science. The approach showed how Opinion mining has greatest interest when used to understand, quantify and apply the sentiment orientation of texts. Moreover that, the proposed analytical methods could be applied in more comprehensive publications to gain more generalized understanding of the structure of a science.

Ouertani and Alawadh (2017) provided a system that is built in the top of a massive open online courses (MOOCs). The provided system improved the learning process for online learner in a way that satisfies the learner 
needs. The system recommends course according to the previous experience of its users (not opinions).

Dharmarajan and Dorairangaswamy (2016) used the FP-growth algorithm for obtaining frequent access patterns from the web log data and providing valuable information about the user's interest. The FP-growth implementation that is used was Rapid Miner Software.

Prasanth (2016) used web usage mining in achieving personalization. Prasanth focused on strategy of automatic web personalization and its various advances. Prasanth (2016) introduced the various advances of web usage mining techniques in the area of web personalization.

Jaskolski et. al. (2016) used Opinion mining in online reviews to enhance the distance education programs. Jaskolski et al. (2016) depended on proposing a multi label hierarchical classification model in which relevant aspects grouped into categories. The aim is to predict both the relevant categories as well as the specific aspects under discussion in a review by using opinion mining and natural language processing. The model implemented in the field of distance education for choosing a program or course to be educated. The model provides satisfactory results on the small or medium corpus. The model provided inaccurate results with large set of data and the results not provided online.

Dong et. al. (2016) described a technique for product recommendation. The technique used not only the features that could be mined from user generated reviews but also the expressions of sentiment that are associated with these features. A recommendation ranking strategy has been provided.

Muhammad et. al. (2016) were interested in studying explanations and how they are playing an important part of modern recommender systems. Muhammad et. al. (2016) said that explanations could help users to make better decisions, improve the conversion rate of browsers into buyers and lead to greater user satisfaction in the long-run. Muhammad et al. (2016) extended the recent work on generating explanations by mining user reviews. Muhammad et.al. (2016) showed how this could lead to a novel explanation format that could be tailored for the needs of the individual user. Moreover Muhammad et. al. (2016) demonstrated how the explanations themselves could be used to rank recommendations, so items which could be associated with a more compelling explanation are ranked ahead of items that have a less compelling explanation. Muhammad et. al. (2016) evaluated their approach using a large-scale, real-world Trip Advisor dataset.

Li et. al. (2015) used text mining method to extract the opinion related information from the massive reviews and analyzed it. A two layer selection algorithm has been designed to find the most suitable products for customers. Li et. al. (2015) experiments were applied on chines sentences. The results showed that the number of word set affected the accuracy of performance of the system. Large numbers decrease the accuracy and efficiency of results.

\section{The Proposed Framework}

Reviewing the literatures showed that most of previous studies depended in building intelligent web on mining and analyzing user's profiles or opinions separately. From the researchers' point of view, this becomes not fair enough. Researchers think that current webs could be more intelligent and efficient if both of user preferences and opinions are considered in building recommendations and enhancements. Depending on user profiles (log files) in providing recommendations becomes not fair enough. Users' profiles aren't reflect one hundred percent the true situation about the user preferable. For example, visiting the page many times not means that user is satisfy or glad with this visit or with page content. User may be has no other choice, like student that must use the page for his online courses to register the term courses. What about the new user? How really he finds the site. Depending only on the previous behaviors couldn't accurately succeed in managing that. All over that, depending only on users' logs waste a vital source of data for any current website. Users' opinions considered now as a crystal part of knowledge that can't be neglected. They reflect what users feel and the level of business success.

On the other hand, systems that depend only on opinions couldn't be considered accurate. Different people have different opinions about the same exact topic or product depending on the purpose for each one.

Researchers try to enhance the recommendations by proposing a framework that could be benefit from the advantages of gained knowledge from mining users' log files and user's reviews. The proposed framework would combine the knowledge gained from both WUM and WOM. The combined gained knowledge from both WUM and WOM would handle the doubtfulness that may be found of each other. Fig 6 shows the proposed framework.

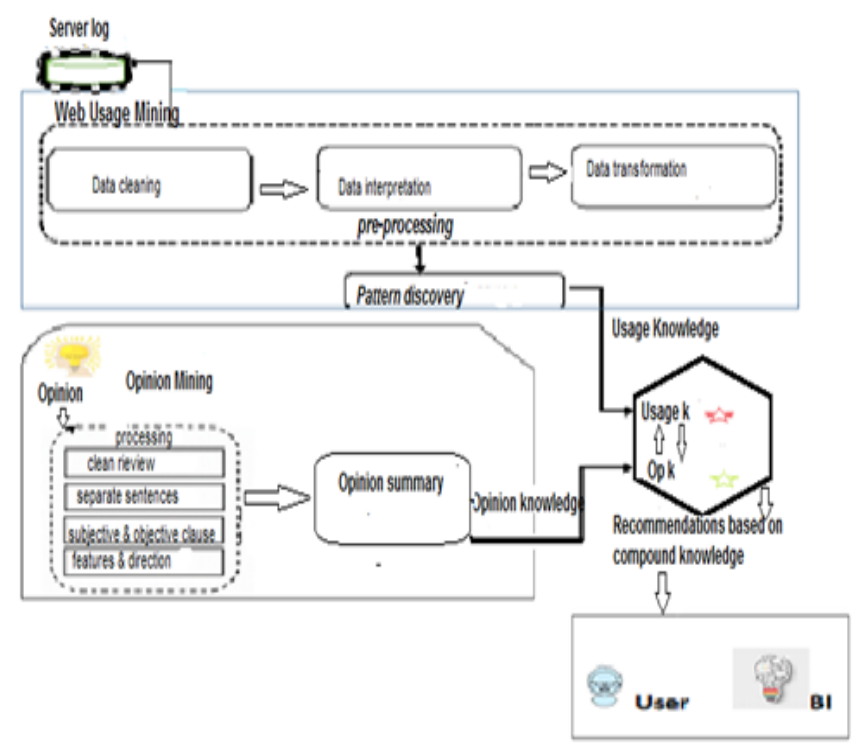

Fig 6. The Proposed Framework

The proposed framework will be applied on an academic website which is Washington University web site. The combined knowledge from the proposed framework will be used in enhancing the site quality. WUM approach used in analyzing the users' site behaviors. The Opinion Mining (OP) approach used in analyzing the opinions of users that uses the site. WUM shows a great knowledge about how the site is being used. Some examples of gained knowledge like what is the most and least requested pages. What is the most used browsers? Which is the most and least referred pages. This knowledge reflects only how the site is being browsed. This could be helpful in site restructure and links rearrangement but to some extent. It is not enough for accurate decisions related to site quality enhancement. Site quality is not limited to the links and how the site is being explored. This is not correct. For example what about the page itself. May be WUM showed that the page has a high frequency visit but it doesn't provide the knowledge about the design of the page itself. How was its content? . Does the user find what he is really looking for? And how?. Was 
it easy? Was it clear?. Is it written in accurate and correct language?. What about links on page, is it well organized?. Is it easy in access?. Does everything in its right place?. What about media, is there any media that could be used to decrease the written part or to show clearly the idea of the page? Many and many questions are needed to be answered with accurate knowledge. These answers form an essential part in taking the site quality enhancement decision. The high frequency of use not mean a good use. User may visit the link several times to do what he wants but this doesn't mean that he is satisfy about this use. OP will handle this shortage by analyzing the opinions of users which actually deal with site. Analyzing users' opinions provide decision maker with the missing knowledge about the entire design of the site. Combining knowledge gained from WUM and OP provide the complete knowledge which is required to take right decisions in the right time. This in turn would reflect on the level of satisfaction of user's site and increases the business performance level. The combined knowledge achieves what the user wants in the right time by precise decisions. This is the intelligence in doing the business.

For the proposed framework experiment, a log file is being used for a WUM purpose and 1023 opinions are mined for the OP purposes. A WEKA and a sentiment analyzer are the mining tools that are used in mining the log and user opinions.

The results showed a set of weak points in the site design. Opinion knowledge demonstrates a set of limitations in web design according to page content, media, ease of access, navigation and the impression about the site as a whole. Figure 7 is an example for exploring general attitude for the users' opinions about Page content presentation (text, image, voice and graphic data). The figure showed a high percentage for a bad attitude (around 49.07\%) about the page content presentation generally for the site. As an example for the gained knowledge from WUM, Table 1 shows a part of knowledge about the most visited pages from table1, the home page is the most high frequency visiting page, but what is the users' opinions about this page?. Figure 8 shows the general attitude about the home page organization and content presentation.
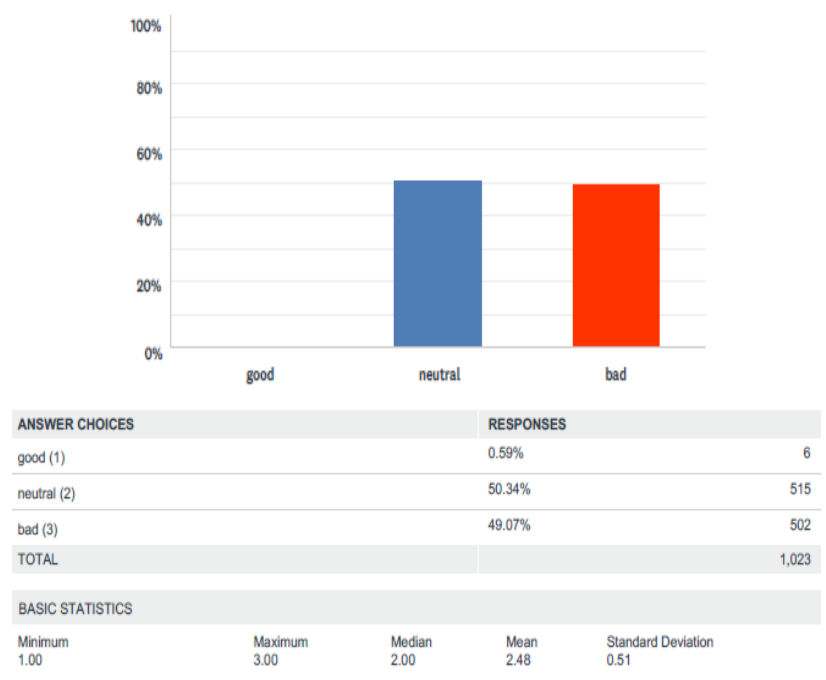

Fig 7. General attitude for the users' opinions about Page content presentation (text, image, voice and graphic data)

\begin{tabular}{|r|l|r|}
\hline & URL & Access \\
\hline 1 & /home/cse.css & 3925 \\
\hline 2 & /education/course-webs.html & 761 \\
\hline 3 & /homes/map/hush/78.html & 718 \\
\hline 4 & /homes/map/hush/dumb.html & 714 \\
\hline 5 & /research/research.html & 532 \\
\hline 6 & $\begin{array}{l}\text { /education/courses/high } \\
\text { graduate.html }\end{array}$ & 497 \\
\hline 7 & /info/current/news.html & 443 \\
\hline 8 & $\begin{array}{l}\text { /education/courses/143/99au/homew } \\
\text { ork/index.html }\end{array}$ \\
\hline
\end{tabular}

Table 1. Part of the most requested pages.

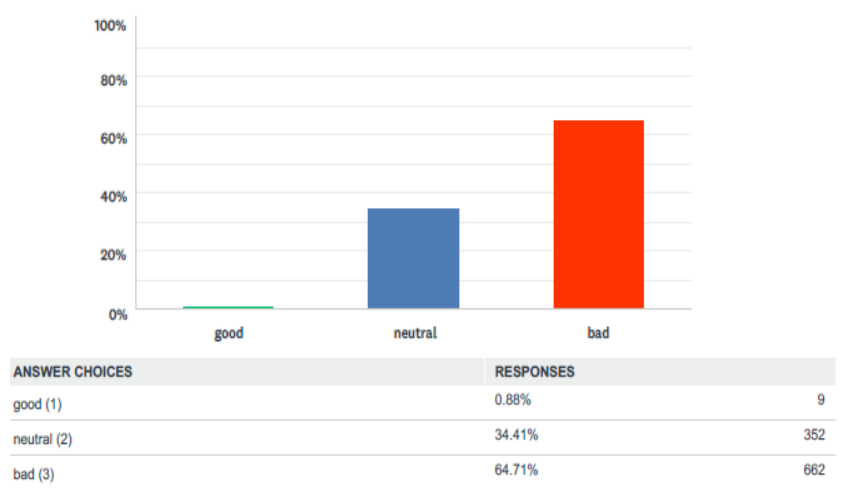

Fig 8. Home page evaluation.

It seems that users have a very bad experience in dealing with the site's homepage. Mining users' opinions showed that more than 64\% (64.71\%) of opinions have a bad experience in dealing with the home page content and design. This means that there is a high necessity to change and improve the design for the page. Analyzing users' opinions not only show the general attitude for the design but it also determines the weak points which are needed to be enhanced. Returning to our example, site's home page, and according to users' opinions, the order of links, labels and titles was the worst drawback point in the home page design. This is a killing mistake especially for any site home page. Home page for any website is the entrance for its services and contents. If the entrance is broken or even cracked it would harm anyone want to use this entrance. A bad homepage gives a very bad impression about the site as a whole. Experiment shows also other points that need to be enhanced like the poor coloring and media use. Figure 9 shows the users' opinions for the order of labels and links.

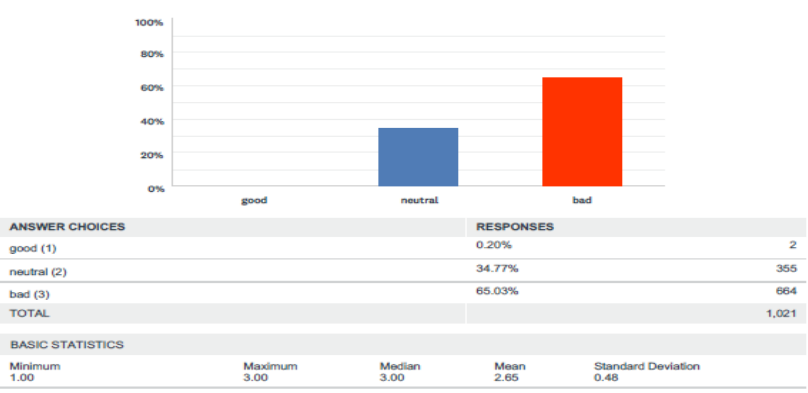

Fig 9. Users' opinions for order of links, labels, titles. 
On the other hand, the results show a strong point in the home page design. The easiness and clearness of writing and writing in correct grammatically way are examples for theses strong points. The results showed a high percentage which is more than $70 \%$ for the clear of writing and more than $69 \%$ for the correct grammar. Figure 10 and figure 11 show these percentages simultaneously.

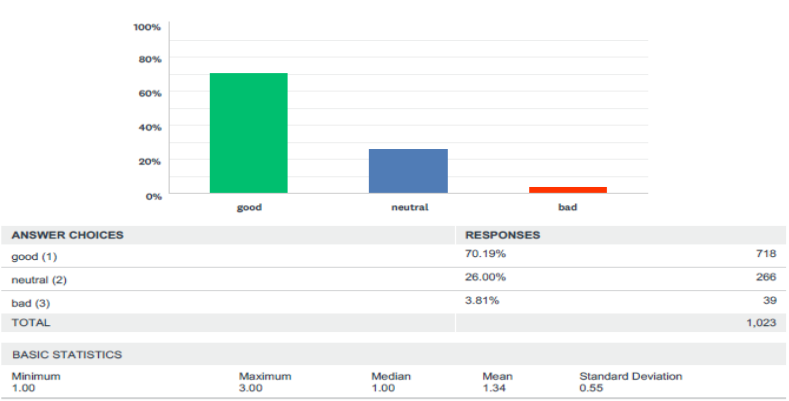

Fig 10. Clearness of writing.

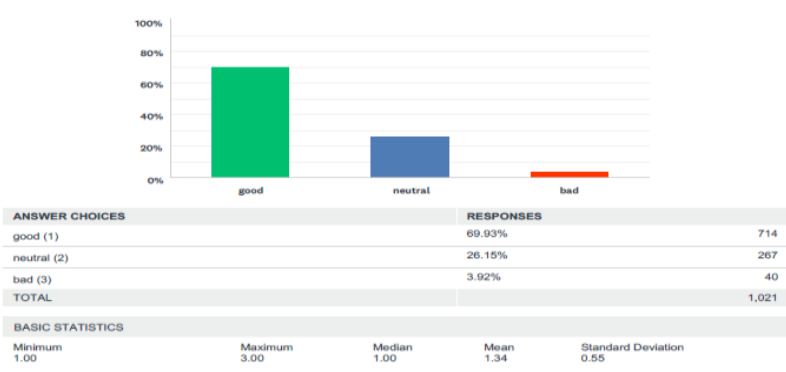

Fig 11. Writing grammatically correct.

\section{Conclusion}

WI presents excellent opportunities and challenges for exploring BI (Yao et al., 2001). Great effect for WI on BI could be touched in the fields like (e-commerce, e-finance, e-science, e-learning, e-government, e-community etc.). Website's logs and opinions are used to capture the behavior of the customer. Opinion mining and Web usage mining analysis play an important role in BI for customer relationship management (CRM) and targeted marketing services.

The paper introduced a proposed framework for WI. The proposed framework depends on combining the knowledge from WUM and OP. The combined knowledge used in achieving the intelligence for the web site. The proposed framework is used in enhancing the quality for the Washington website university. The experiment showed good results that proof a high confidence in the proposed framework. The quality issue has been chosen by the study to be tested by a proposed framework. Site's quality is considered a central factor for achieving the WI. In WI it is important to deliver what the user wants or interests but it is more important how to deliver it. Site's quality insures the quality in providing service or content. Gaining the user's high satisfaction means successful in achieving the BI application.

A background review and literature survey toward using WI in enhancing BI applications also provided. The growing popularity of social and EBusiness sites made the WI could be seen as the heart process in succeeding the BI. First part of the paper (background) presents a view for WI and BI followed by the topics which are included in moving to WI. The paper tried to concentrate in each topic on the main points which could be useful in understanding it. The topics included are Recommender System (RS), Web Opinion Mining (WOM) and Web Usage Mining (WUM).

In the Second part, the paper explored and provided a wide range of researches in the field of WI and BI. This could be used as a guide to benefit from the previous work in this field. Exploring those researches, showed that depending only on user preferences in recommendations was sufficient. Many researches illustrated the benefits from using user's preferences in enhancing the quality of services that provided by a site. The web intelligence at this stage was depending only on the former history of user navigation like in (Dharmaraian \& Dorairangaswamy, 2016; Doja, 2017; Prasanth, 2016; Pushpalatha \& Chandra, 2017). Thinking in that approach (intelligence based on user preferences), showed some limitations. For example the new user was not be considerable. How could he benefits from the intelligence of the site. New user with no preferences, what kind of recommendations could be presented to him and to what extend the accuracy of recommendations. Another issue, depending only on user preferences neglect a vital source of information which is user opinions. User opinions form an essential part of any website. Especially in the ecommerce field which highly depends on the views (opinions) of the customers and benefit from using web as a crystal interface for their enterprises. Attention to the value of user opinions made a great leap toward the approach of opinion mining and how it could enhance the web intelligence. Many researches used opinions from different points of views and in different fields as (Yuan et al., 2017) succeeded in using a sophisticated kind of opinions which is emotional features and social interactions to provide recommendations that meet user needs, ( Balahadia, $\&$ Comendador, 2016) depended on opinions in enhancing the performance and the level of services provided by a faculty and ( Jaskolski et al.,2016) used opinion mining in predicting the relevant categories as well as the specific aspects in the field of distance education .

Thinking in depending only on opinions in providing recommendations might be imprecise. Opinions not always provide the truth. As we said before, different peoples have different opinions about the same exact product or service depending on their target.

The third part of the paper introduced the proposed framework which combine the knowledge gained from WUM and WOM. From the experiment, results shed the light clearly on the importance of the proposed framework idea. Combining the WUM and OP knowledge services as a strong backbone or base stone in taking a decisions for enhancing the site quality. The combined knowledge presents a clear image about the actual situation. As it has been shown, a home page as an example and according to WUM knowledge, it is ok and no enhancement is needed. OP knowledge showed a revised image. OP results recommended highly that It should be enhanced in it is organization and presentation for data. Most opinions (more than 60\%) said that it is bad and not sufficient. Mining opinions not only show the limitation presences but also show where and which the weak points are and focus on it. 


\section{REFERENCES}

Adomavicius, G., \& Tuzhilin, A. (2005). Toward the next generation of recommender systems: A survey of the state-of-the-art and possible extensions. IEEE transactions on knowledge and data engineering, 17(6), 734-749.

Agrawal, R., \& Srikant, R. (1994, September). Fast algorithms for mining association rules. In Proc. 20th int. conf. very large data bases, $V L D B$ (Vol. 1215, pp. 487-499).

Agrawal, R., Imieliński, T., \& Swami, A. (1993, June). Mining association rules between sets of items in large databases. In Acm sigmod record (Vol. 22, No. 2, pp. 207-216). ACM.

Ahuja, S. (2017). Using the Flipped Classroom to Improve Knowledge Creation of Master's-Level Students in Engineering. In Handbook of Research on Instructional Systems and Educational Technology (pp. 326339). IGI Global.

Balahadia, F. F., \& Comendador, B. E. V. (2016). Adoption of Opinion Mining in the Faculty Performance Evaluation System by the Students Using Naïve Bayes Algorithm. International Journal of Computer Theory and Engineering, 8(3), 255.

Bobadilla, J., Ortega, F., Hernando, A., \& Gutiérrez, A. (2013). Recommender systems survey. Knowledge-based systems, 46, 109-132.

Burke, R. (2002). Hybrid recommender systems: Survey and experiments. User modeling and user-adapted interaction, 12(4), 331-370.

Camacho, D. (2013, June). Challenges and issues of web intelligence research. In Proceedings of the 3rd International Conference on Web Intelligence, Mining and Semantics (p. 0). ACM.

Candillier, L., Meyer, F., \& Boullé, M. (2007, July). Comparing state-of-theart collaborative filtering systems. In International Workshop on Machine Learning and Data Mining in Pattern Recognition (pp. 548-562). Springer, Berlin, Heidelberg.

Dharmarajan, K., \& Dorairangaswamy, D. M. (2016). Web Usage Mining: Improve The User Navigation Pattern Using Fp-Growth Algorithm. Elysium journal of engineering research and management (EJERM), 3(4).

Doja, M. N. (2017). Web Usage Mining Techniques to Improve the Capabilities of E-learning Websites and Blogs. International Journal of Advanced Research in Computer Science, 8(5).

Dong, R., \& Smyth, B. (2016, October). Personalized Opinion-Based Recommendation. In International Conference on Case-Based Reasoning (pp. 93-107). Springer International Publishing.

Dong, R., O’Mahony, M. P., Schaal, M., McCarthy, K., \& Smyth, B. (2016). Combining similarity and sentiment in opinion mining for product recommendation. Journal of Intelligent Information Systems, 46(2), 285312

Ekstrand, M. D., Riedl, J. T., \& Konstan, J. A. (2011). Collaborative filtering recommender systems. Foundations and Trends ${ }^{\circledR}$ in Human-Computer Interaction, 4(2), 81-173.

Funk, A., Li, Y., Saggion, H., Bontcheva, K., \& Leibold, C. (2008, October). Opinion analysis for business intelligence applications. In Proceedings of the first international workshop on Ontology-supported business intelligence (p. 3). ACM.

Gonsalves, T. (2015). The State of the Art in Web Mining. In Encyclopedia of Information Science and Technology, Third Edition (pp. 1937-1947). IGI Global.

Herlocker, J. L., Konstan, J. A., Terveen, L. G., \& Riedl, J. T. (2004). Evaluating collaborative filtering recommender systems. ACM Transactions on Information Systems (TOIS), 22(1), 5-53.

Jaskolski, J., Siegberg, F., Tibroni, T., Cimiano, P., \& Klinger, R. (2016). Opinion Mining in Online Reviews About Distance Education Programs. arXiv preprint arXiv:1607.06299.

Kim, Y., \& Jeong, S. R. (2015). Opinion-Mining Methodology for Social Media Analytics. KSII Transactions On Internet \& Information Systems, 9(1)
Konstan, J. A., \& Riedl, J. (2012). Recommender systems: from algorithms to user experience. User Modeling and User-Adapted Interaction, 22(1), 101123.

Krulwich, B. (1997). Lifestyle finder: Intelligent user profiling using largescale demographic data. AI magazine, 18(2), 37.

Lang, K. (1995, July). Newsweeder: Learning to filter netnews. In Proceedings of the 12th international conference on machine learning (Vol. 10, pp. 331-339).

Laursen, G. H., \& Thorlund, J. (2016). Business analytics for managers: Taking business intelligence beyond reporting. John Wiley \& Sons.

Leat, V. (2007). Introduction to business intelligence. IBM Software Group, raspoloživo na: http://www07. Ibm. Com/sg/events/blueprint/pdf/day1/Introduction_to_Business_Intelligence. pdf,[Pristupljeno 07.11. 2016].

Li, X., Wang, H., \& Yan, X. (2015). Accurate recommendation based on opinion mining. In Genetic and Evolutionary Computing (pp. 399-408). Springer, Cham.

Liu, B. (2012). Sentiment analysis and opinion mining. Synthesis lectures on human language technologies, 5(1), 1-167.

Liu, B., Mobasher, B., \& Nasraoui, O. (2011). Web usage mining. In Web Data Mining (pp. 527-603). Springer Berlin Heidelberg.

Marmanis, H., \& Babenko, D. (2009). Algorithms of the intelligent web (pp. 69-120). Greenwich: Manning.

Mithaiwala, N. J., \& Jariwala, N. B. (2016, May). A Study on Web Intelligence: Trends, Challenges and Future. In National Conference on Recent Trends in Computer and Communication Technology (Vol. 10, p. 11).

Muhammad, K., Lawlor, A., \& Smyth, B. (2016, March). On the Use of Opinionated Explanations to Rank and Justify Recommendations. In FLAIRS Conference (pp. 554-559).

Neelima, G., \& Rodda, S. (2015). An overview on web usage mining. In Emerging ICT for Bridging the Future-Proceedings of the 49th Annual Convention of the Computer Society of India CSI Volume 2 (pp. 647-655). Springer International Publishing.

Ouertani, H. C., \& Alawadh, M. M. (2017). MOOCs Recommender System: A Recommender System for the Massive Open Online Courses. In Innovations in Smart Learning (pp. 137-140). Springer Singapore.

Paulson, P., \& Tzanavari, A. (2003). Combining collaborative and contentbased filtering using conceptual graphs. Modelling with Words, 168-185.

Pazzani, M. J. (1999). A framework for collaborative, content-based and demographic filtering. Artificial intelligence review, 13(5-6), 393-408.

Popovič, A., Hackney, R., Coelho, P. S., \& Jaklič, J. (2012). Towards business intelligence systems success: Effects of maturity and culture on analytical decision making. Decision Support Systems, 54(1), 729-739.

Porcel, C., Tejeda-Lorente, A., Martínez, M. A., \& Herrera-Viedma, E. (2012). A hybrid recommender system for the selective dissemination of research resources in a technology transfer office. Information Sciences, 184(1), 119.

Prasanth, A. (2016). Web Personalization using Web Usage Mining Techniques. International Journal of Current Engineering and Scientific Research, 3(3), 45-49.

Pushpalatha, N., \& Chandra, N. S.(2017). An Extensible Web Mining Framework for Real Knowledge.

Ranjan, J. (2009). Business intelligence: Concepts, components, techniques and benefits. Journal of Theoretical and Applied Information Technology, 9(1), 60-70.

Rao, K. N. (2008). Application domain and functional classification of recommender systems--a survey. DESIDOC Journal of Library \& Information Technology, 28(3), 17.

Reinschmidt, J., \& Francoise, A. (2000). Business intelligence certification guide. IBM International Technical Support Organisation.

Savitha, S. J., Fernando, D. B. S., \& Saranya, K.(2017). Building a Scalable Eservice Recommender System. International Journal of Computer Trends and Technology (IJCTT) - Volume 46 Number 1- April 2017.

Scholz, M., Dorner, V., Schryen, G., \& Benlian, A. (2017). A configurationbased recommender system for supporting e-commerce decisions. European Journal of Operational Research, 259(1), 205-215. 
Sharma, R., Nigam, S., \& Jain, R. (2014). Opinion mining in Hindi language: a survey. arXiv preprint arXiv:1404.4935.

Suharjito, S. S. (2016). Implementation of Classification Technique in Web Usage Mining of Banking Company. 2016 International Seminar on Intelligent Technology and Its Applications (ISITIA).IEEE.

Sun, X., Kong, F., \& Ye, S. (2005, March). A comparison of several algorithms for collaborative filtering in startup stage. In Networking, Sensing and Control, 2005. Proceedings. 2005 IEEE (pp. 25-28). IEEE.

Symeonidis, P., Nanopoulos, A., \& Manolopoulos, Y. (2009, October). MoviExplain: a recommender system with explanations. In Proceedings of the third ACM conference on Recommender systems (pp. 317-320). ACM.

Van Meteren, R., \& Van Someren, M. (2000, May). Using content-based filtering for recommendation. In Proceedings of the Machine Learning in the New Information Age: MLnet/ECML2000 Workshop (pp. 47-56).

Yao, Y. Y., Zhong, N., Liu, J., \& Ohsuga, S. (2001). Web Intelligence (WI) research challenges and trends in the new information age. In Web intelligence: Research and development (pp. 1-17). Springer, Berlin, Heidelberg.
Yuan, S. T., Yuan, S. T., Yang, C. Y., \& Yang, C. Y. (2017). Service recommender system based on emotional features and social interactions. Kybernetes, 46(2), 236-255.

Zhu, Y., Kim, M. C., \& Chen, C. (2017). An investigation of the intellectual structure of opinion mining research. Information Research, 22(1) 